\title{
Use of antimicrobials for animals in New Zealand, and in comparison with other countries
}

\section{JE Hillerton ${ }^{* \&_{1}}$, CR Irvine ${ }^{\dagger}$, MA Bryan ${ }^{\ddagger}$, D Scott ${ }^{\#}$ and SC Merchant ${ }^{\sharp}$}

*Drumlanrig, 163 Flume Road, RD4, Cambridge 3496, New Zealand

${ }^{\dagger}$ New Zealand Veterinary Association, PO Box 11212, Wellington 6011, New Zealand

"VetSouth, Cnr Great North and Welsh Roads, PO Box 12, Winton, New Zealand

"Ethical Agents, 470 Parnell Road, Auckland, New Zealand

${ }^{\ddagger}$ Pet Doctors, 743 Mount Eden Road, Mount Eden, Auckland 1024, New Zealand

${ }^{\S}$ Author for correspondence: Email: hillerton@ netsmart.net.nz

Supplementary Table 1. Data sources and methods of calculation for antimicrobial use based on sales, animal and human populations, and weight classes.

The content of this supplementary information has not been edited. All risk and liability rest with the authors. 
Supplementary Information

Animals

\section{Antimicrobials}

\section{European countries}

ESVAC publication ${ }^{\text {a }}$ using data from wholesalers pharmacies and clinics

Australia

2009-2010 data from Australian Pesticides and Veterinary Medicines

Authority

\section{Canada}

Public Health Agency of Canadak $^{\mathrm{k}}$, Canadian antimicrobial resistance surveillance system

\section{New Zealand}

Ministry of Primary Industries, Agricultural Chemicals and Veterinary Medicines reports ${ }^{\text {pqr }}$

USA

Food and Drug

Administration, Summary report on antimicrobials sold or distributed for use in food-producing animals ${ }^{u}$ Agriculture census ${ }^{v}$
Populations: Australian Bureau of Statistics, Agricultural Commodities Weights: Department of Agriculture and industry levy-body databases ${ }^{g h}$ see Supp. Table 2

Populations: Canadian agricultural census 2012

Weights: Used US data, Land grant university web sites, see Supp.

Table 2

\section{Populations: Statistics New} Zealand, Agricultural tables

livestock numbers'; horses S

Cooper, Horse Racing Board pers.

comm.; cats and dogs, New

Zealand Companion Animal Council

Weights: Industry levy-body web

sites, publications and their

veterinarians, See Supp. Table 2

\section{Populations: US Department of}

Weights: Land grant university web sites, see Supp. Table 2
Calculation(s)

Values taken directly from ESVAC publication ${ }^{a}$ which used the

European Medicines Authority (EMA) method ${ }^{c}$

Made by authors using the EMA method and from Australia's first

national antibiotic

resistance strategy

Made by authors using the EMA method

Government of Canada Human antimicrobial use report $^{\mathrm{m}}$

Made by authors using the EMA method

Ministry of Health via Dr MG Thomas and Dr A Smith pers. comm.

\section{Made by authors using} the EMA method

Food and Drug Administration,

Antibacterial drug use analysis ${ }^{\mathrm{w}}$

publication ${ }^{d}$ using

data from

\section{Humans}

Biomass

ECDC/EFSA/EMA publication

pharmacies and

\section{OECD Health statistics 2013 \\ OECD Health statistics 2013}

\section{Author extrapolation} from OECD' by comparison with ECDC/EFSA/EMA data for similar use rate

Population: Statistics Canada

Weights: guidance

data for health based calculations, EFSA ${ }^{\circ}$

Population: Statistics New Zealand,

census

Weights: guidance

data for health based calculations, EFSA

\section{Population: US} census, $2012^{\mathrm{x}}$ Weights: guidance

data for health based calculations, EFSA ${ }^{\circ}$, US census ${ }^{x}$
Made by authors using

Made by authors using the EMA method

Made by authors using the EMA method

the EMA method 
${ }^{\text {a }}$ Anonymous. Sales of Veterinary Antimicrobial Agents in 26 EU/EEA Countries in 2012

http://www.ema.europa.eu/docs/en GB/document library/Report/2014/10/WC500175671.pdf (accessed 14 March 2016). European Medicines Agency, London, UK, 2014

${ }^{\mathrm{b}}$ Montforts MHMM. Environmental risk assessment for veterinary medicinal products. Part 1. Other than GMO-containing and immunological products. First update http://nl.sitestat.com/rivm/rivm-

nl/s?link.en.documents and publications.scientific.reports.1999.april.environmental risk assessment for veterinary medicinal products part 1 other than $\mathrm{g}$ mo containing and immunological products first update.download pdf\&ns type=pdf\&ns url=http\%3A\%2F\%2Fwww.rivm.nl\%2Fdsresource\%3Fobjectid=rivm p:15550\&type=org\&disposition=inline\&ns $\mathrm{nc}=1$ (accessed 14 March 2016). National Institute for Public Health and the Environment (RIVM), Bilthoven, The Netherlands, 1999

${ }^{c}$ Anonymous. Trends in the sales of veterinary antimicrobial agents in nine European countries (2005-2009) 'http://www.ema.europa.eu/docs/en GB/document library/Report/2011/09/WC500112309.pdf (accessed 14 March 2016). European Medicines Agency, London, UK, 2011

${ }^{\mathrm{d}}$ Anonymous. ECDC/EFSA/EMA first joint report on the integrated analysis of the consumption of antimicrobial agents and occurrence of antimicrobial resistance in bacteria from humans and food-producing animals. EFSA Journal 13, 4006, doi:10.2903/j.efsa.2015.4006, 2015

${ }^{\text {e }}$ Anonymous. Quantity of Antimicrobial Products Sold for Veterinary Use in Australia: July 2005 to June 2010. http://archive.apvma.gov.au/publications/reports/docs/antimicrobial sales report march-2014.pdf (accessed 14 March 2016). Australian Pesticides and Veterinary Medicines Authority, Kington, ACT, Australia, 2014

${ }^{\dagger}$ Anonymous. Agricultural Commodities, Australia, 2009-10 http://www.abs.gov.au/AUSSTATS/abs@.nsf/DetailsPage/7121.02009-10?OpenDocument (accessed September 2015). Australian Bureau of Statistics, Sydney, Australia, 2011

${ }^{9}$ Gordon J. The Horse Industry. Contributing to the Australian economy https://rirdc.infoservices.com.au/downloads/01-083 (accessed 14 March 2016). Rural Industries Development Corporation, Barton ACT, Australia , 2001 


\section{Supplementary Information}

${ }^{\text {n}}$ Shapiro S. Deer Industry Database https://rirdc.infoservices.com.au/downloads/09-175 (accessed 14 March 2016). Rural Industries Development Corporation, Barton ACT, Australia, 2010

'Anonymous. Responding to the Threat of Antimicrobial Resistance: Australia's First National Antimicrobial Resistance Strategy 2015-2019 http://www.health.gov.au/internet/main/publishing.nst/Content/1803C433C71415CACA257C8400121B1F/\$File/amr-strategy-2015-2019.pdf (accessed 14 March 2016). Australian Government, Canberra, ACT, Australia, 2015

${ }^{j}$ Anonymous. Government at a Glance 2015 P 185 http://dx.doi.org/10.1787/888933249573 (accessed 24 March 2016), OECD Publishing, Paris, France, 2015

${ }^{\mathrm{k}}$ Anonymous. Canadian antimicrobial resistance surveillance system-report 2015 http://healthycanadians.gc.ca/alt/pdf/publications/drugs-products-medicamentsproduits/antibiotic-resistance-antibiotique/antimicrobial-surveillance-antimicrobioresistance-eng.pdf (accessed 14 March 2016). Public Health Agency of Canada, Ottawa, Canada, 2015

' Anonymous. Statistics Canada 2012 Agricultural census http://www.statcan.gc.ca/pub/12-581-x/2012000/agri-eng.htm (accessed 30 September 2015). Statistics Canada, Ottawa, Canada, 2015

${ }^{m}$ Anonymous. Human Antimicrobial Use Report, 2012/2013. http://www.phac-aspc.gc.ca/publicat/hamdur-rumamh/2012-2013/index-eng.php (accessed 30 September 2015), Public Health Agency of Canada, Guelph, Canada, 2015

${ }^{n}$ Anonymous. Statistics Canada, Census 2012. http://www.statcan.gc.ca/tables-tableaux/sum-som/101/cst01/demo02a-eng.htm (accessed 30 September 2015). Statistics Canada, Ottawa, Canada, 2015

${ }^{\circ}$ Anonymous. Guidance on selected default values to be used by the EFSA Scientific Committee, Scientific Panels and Units in the absence of actual measured data. European Food Safety Authority Journal 10:2579-2611, 2012

${ }^{\mathrm{p}}$ Anonymous. Antibiotic Sales and Use Overview 2004-2009 www.foodsafety.govt.nz/elibrary/industry/antibiotic-sales-2004-2009.pdf (accessed 14 March 2016) Ministry of Agriculture and Forestry, Wellington, NZ, 2010

${ }^{9}$ Anonymous. Antibiotics Sales Analysis: 2009-2011, MPI Technical Paper No: 2013/62 http://www.foodsafety.govt.nz/elibrary/industry/report-antibiotics-sales2009-2011.pdf (accessed 14 March 2016). Ministry for Primary Industries, Wellington, NZ, 2012 


\section{Supplementary Information}

${ }^{\top}$ Anonymous. Antibiotic Sales Analysis: 2011-2014, Ministry for Primary Industries, Wellington, NZ, unpublished data

${ }^{s}$ Anonymous. Livestock Numbers by Regional Council http://nzdotstat.stats.govt.nz/wbos/Index.aspx?DataSetCode=TABLECODE7423 (accessed 9 September 2015), Statistics New Zealand, Wellington, New Zealand, 2015

${ }^{\mathrm{t}}$ Anonymous. Subnational population estimates http://nzdotstat.stats.govt.nz/wbos/Index.aspx?DataSetCode=TABLECODE7502\# (accessed 9 September 2015), Statistics New Zealand, Wellington, New Zealand, 2015

${ }^{u}$ Anonymous. 2012 Summary Report on Antimicrobials Sold or Distributed for Use in Food-Producing Animals http://www.fda.gov/downloads/Forlndustry/UserFees/AnimalDrugUserFeeActADUFA/UCM416983.pdf (accessed 9 September 2015), Food and Drug Administration, MD, USA

${ }^{\vee}$ Anonymous. 2012 Census of Agriculture http://www.agcensus.usda.gov/Publications/2012/Full Report/Volume 1, Chapter 1 US/ (accessed 9 September 2015). United States Department of Agriculture, MD, USA, 2012

${ }^{\text {w }}$ Anonymous. Antibacterial Drug Use Analysis www.fda.gov/downloads/drugs/drugsafety/informationbydrugclass/ucm319435.pdf (accessed 9 September 2015). US Food and Drug Administration, .MD, USA, 2012

${ }^{x}$ Anonymous. Age and Sex Composition in the United States: 2012 http://www.census.gov/population/age/ (accessed 9 September 2015), US Census Bureau, MD, USA 
Supplementary Information

Supplementary Table 2. Data, and their sources, used to calculate population correction units (Biomass). See also data sources in Supplementary Table 1.

Species

$$
\text { Age/gender }
$$

Average weight (kg)

Comments

Data sources

New Zealand ${ }^{a}$

Beef cattle

0-1 year $\quad 225$

1-2 years $\quad 320$

$>2$ years $\quad 500$

Dairy Cattle

0-1 year

1-2 years

$>2$ years

Sheep

Hoggets

56

Ewes + rams

63

Deer

$<1$ year

95

Mature

Pigs
Estimated 6 month weight over all breeds

Estimated 18 month weight over all

breeds

Includes bulls for dairy, e.g. Jersey

Estimated 6 month weight over all breeds

Estimated 18 month weight over all breeds

Takes account of proportion of herd of

Jersey, Kiwi cross and Friesian

Beef+Lamb New Zealand, C. Houston

Dr A. Bell, deer veterinarian

NZPork, F. Clements

Beef+Lamb New Zealand, C. Houston

DairyNZ, K. Macdonald 
Supplementary Information

\begin{tabular}{|c|c|c|c|c|}
\hline Species & Age/ gender & Average weight (kg) & Comments & Data sources \\
\hline \multicolumn{5}{|l|}{ New Zealand ${ }^{a}$} \\
\hline \multicolumn{5}{|l|}{ Poultry } \\
\hline & Layers & 2.3 & $\begin{array}{l}\text { Same breeds and production system. } \\
\text { Others include turkeys, ducks and geese }\end{array}$ & PIANZ and USA data \\
\hline & Broilers & 0.99 & & \\
\hline & Other & 1.97 & & \\
\hline \multicolumn{5}{|l|}{ Goats } \\
\hline & Does & 59 & $\begin{array}{l}\text { Bucks too few and too similar to mature } \\
\text { does to make a difference }\end{array}$ & $\begin{array}{l}\text { Dr V McLean, Dairy Goat } \\
\text { Cooperative }\end{array}$ \\
\hline \multicolumn{5}{|l|}{ Horses } \\
\hline & All & 500 & $\begin{array}{l}\text { Estimate for a very wide range of horse } \\
\text { types }\end{array}$ & Horse Racing Levy Board \\
\hline \multicolumn{5}{|c|}{ Australia, Canada, USA ${ }^{\mathrm{b}}$} \\
\hline \multicolumn{5}{|c|}{ Beef Cattle } \\
\hline & $0-1$ year & 180 & Estimated 6 month weight over all breeds & $\begin{array}{l}\text { Statistics bodies, university } \\
\text { extension services, broad } \\
\text { literature search often of } \\
\text { promotional material. }\end{array}$ \\
\hline & $1-2$ years & 480 & $\begin{array}{l}\text { Estimated } 18 \text { month weight over all } \\
\text { breeds }\end{array}$ & \\
\hline & $>2$ years & 590 & Includes bulls for dairy. & \\
\hline \multicolumn{5}{|c|}{ Dairy Cattle } \\
\hline & $0-1$ year & 406 & Estimated 6 month weight over all breeds & $\begin{array}{l}\text { Statistics bodies, university } \\
\text { extension services. }\end{array}$ \\
\hline & $1-2$ years & 300 & $\begin{array}{l}\text { Estimated } 18 \text { month weight over all } \\
\text { breeds }\end{array}$ & \\
\hline & $>2$ years & 680 & $\begin{array}{l}\text { Takes account of proportion of herd } \\
\text { breeds. }\end{array}$ & \\
\hline
\end{tabular}


Supplementary Information

\begin{tabular}{|c|c|c|c|c|}
\hline Species & Age/gender & Average weight (kg) & Comments & Data sources \\
\hline \multicolumn{5}{|c|}{ Australia, Canada, USA ${ }^{b}$} \\
\hline \multicolumn{5}{|c|}{ Sheep } \\
\hline & Hoggets & 45 & & $\begin{array}{l}\text { Statistics bodies, university } \\
\text { extension services. }\end{array}$ \\
\hline & Ewes + rams & 60 & & \\
\hline \multicolumn{5}{|c|}{ Deer/elk/bison } \\
\hline & $<1$ year & 70 & & Dr A. Bell, deer veterinarian \\
\hline & $1-2$ years & 100 & & \\
\hline & $>2$ years & 120 & & \\
\hline \multicolumn{5}{|l|}{ Pigs } \\
\hline & All young stages & 55 & & USDA statistics data \\
\hline & Gilts & 100 & & \\
\hline & Sows and boars & 180 & & \\
\hline \multicolumn{5}{|l|}{ Poultry } \\
\hline & Layers & 2.3 & $\begin{array}{l}\text { Same breeds and production system. } \\
\text { Others include ducks and geese }\end{array}$ & $\begin{array}{l}\text { US National Chicken Council } \\
\text { and USDA statistics data }\end{array}$ \\
\hline & Broilers & 0.99 & & \\
\hline & Turkeys & 3.6 & & \\
\hline & Other & 2 & & \\
\hline \multicolumn{5}{|l|}{ Goats } \\
\hline & Does & 59 & $\begin{array}{l}\text { Bucks too few and too similar to mature } \\
\text { does to make a difference }\end{array}$ & $\begin{array}{l}\text { New Zealand estimate } \\
\text { compared with Supp. Table } \\
1 \text { sources }\end{array}$ \\
\hline \multicolumn{5}{|l|}{ Horses } \\
\hline & All & 500 & $\begin{array}{l}\text { Estimate for a very wide range of horse } \\
\text { types }\end{array}$ & $\begin{array}{l}\text { New Zealand estimate } \\
\text { compared with Supp. Table } \\
1 \text { sources }\end{array}$ \\
\hline
\end{tabular}

\footnotetext{
${ }^{a}$ These values reflect the more extensive systems common in New Zealand. The age ranges and animal types are used by Statistics New Zealand and/or the industry levy bodies.

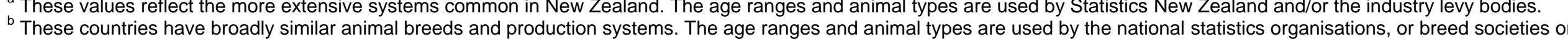
university extension services.
} 